\title{
Evaluación de la efectividad de un programa piloto de optimización del uso de los recursos de Biblioteca en la Universidad San Sebastián y de la satisfacción de los estudiantes, basado en el cambio actitudinal de 18 académicos*
}

\author{
Effectiveness Assessment of Use Optimization Pilot Program of Library Resources \\ in the University San Sebastian and Satisfaction of Students, Based on Attitudinal \\ Change in 18 professors
}
Avaliação de eficácia de um projeto-piloto de otimização de recursos de biblioteca na Universidade San Sebastian e satisfação dos alunos, com base na mudança de atitude em 18 professores

\author{
Luis González Bravo, ${ }^{a}$ Gonzalo Puentes Soto, ${ }^{b}$ Javier Vera Junemann, ${ }^{c}$ \\ Leoncio Esmar Gutiérrez, ${ }^{d}$ José Venegas Lillo, ${ }^{e}$ Marcela Moroni López, ${ }^{f}$ \\ Kiyoshi Fukushi Mandiola, ${ }^{g}$ Erwin Vega Venegas, ${ }^{h}$ Maruzzella Valdivia Peralta ${ }^{i}$ \\ a Escuela de Psicología, Universidad San Sebastián. Lientur 1457, Piso 3, Edificio Los Notros, \\ Concepción, Chile. Fono: 41-2400174. Correo electrónico: luis.gonzalezb@uss.cl \\ b Universidad San Sebastián. Fono: 02-6613190. Correo electrónico: gonzalo.puentes@uss.cl \\ c Universidad San Sebastián. Fono: 41-2400100. Correo electrónico: javier.vera@uss.cl \\ d Universidad San Sebastián. Fono: 02-6613241. Correo electrónico: leoncio.esmar@uss.cl \\ e Universidad San Sebastián. Fono: 02-6614795. Correo electrónico: jose.venegas@uss.cl \\ ${ }^{\mathrm{f}}$ Universidad San Sebastián. Fono: 41-2400302. Correo electrónico: marcela.moroni@uss.cl \\ g Universidad Gabriela Mistral. Fono: 02-24144538. Correo electrónico: kiyoshi.fukushi@ugm.cl \\ ${ }^{\text {h } U n i v e r s i d a d ~ S a n ~ S e b a s t i a ́ n . ~ F o n o: ~ 02-6614718 . ~ C o r r e o ~ e l e c t r o ́ n i c o: ~ e r w i n . v e g a @ u s s . c l ~}$ \\ ${ }^{i}$ Universidad de Concepción. Fono: 41-2204214. Correo electrónico: mavaldivia@udec.cl
}

\begin{abstract}
RESUMEN
Se evalúa la efectividad de un programa piloto en la Universidad San Sebastián orientado a aumentar la satisfacción de los estudiantes con los recursos disponibles en biblioteca mediante la implementación de un cambio actitudinal en los profesores para la sugerencia de textos. Se compararon los grados de satisfacción en siete indicadores entre una muestra por accesibilidad de 175 estudiantes de 18 asignaturas, y una muestra aleatoria de estudiantes participantes en los estudios de Calidad de Servicio 2010 y 2011. Se encontró un mayor nivel de satisfacción en todos los indicadores, siendo significativos los aumentos en cinco variables en comparación con el año 2010, y en cuatro variables en comparación con el 2011. Finalmente, se discuten implicancias y limitaciones de los hallazgos.

Palabras clave: recursos de biblioteca, satisfacción de estudiantes, académicos.
\end{abstract}

* Agradecemos al Comité Ejecutivo de la Sede Concepción, a la Directora de Bibliotecas USS Señorita Pamela Menay y a todo su equipo, así como al Señor Roberto de la Vega por su constante apoyo en esta iniciativa. 


\section{ABSTRACT}

The present research assesses the effectiveness of a pilot program in San Sebastian University to increase the students' satisfaction with the library available resources, through the implementation of an attitudinal change in the courses' professors toward suggesting texts. Satisfaction levels in seven indicators was compared between an accessibility sample of 175 students of 18 pilot courses, and a random sample of students participating in the quality of service studies 2010 and 2011. A greater level of satisfaction in all indicators was found. Five of them were significant with the 2010 comparison, and four of them with the 2011 comparison. Finally, implications and limitations of the study are discussed.

Key words: library resources, students' satisfaction, faculty.

\section{RESUMO}

Objetivou-se avaliar a eficácia de um programa piloto na Universidade San Sebastián a fim de aumentar a satisfação de alunos com os recursos disponíveis na biblioteca, por meio da implementação de uma mudança atitudinal nos professores de cursos para textos que sugerem. Níveis de satisfação em sete indicadores foram comparados em uma amostra por acessibilidade em 175 alunos de 18 cursos-piloto e em uma amostra aleatória de estudantes que participam nos estudos da qualidade do serviço 2010 e 2011 . Verificou-se um maior nível de satisfação em todos os indicadores. Cinco deles foram significativos em comparação a 2010 e quatro deles, em comparação a 2011. Finalmente, discutem-se implicações e limitações do estudo.

Palavras chave: recursos da biblioteca, satisfação de alunos, professores.

\section{MARCO TEÓRICO}

A pesar de que existe un consenso generalizado en lo imprescindible que es disponer de adecuados sistemas de biblioteca en las universidades, y de que los académicos estimulen a sus estudiantes a usarlos, llama la atención la escasa evidencia que existe en la literatura en castellano con respecto al tema.

Los procesos de enseñanza-aprendizaje se fortalecen en la medida que se utilizan efectivamente los recursos de biblioteca, en un alineamiento eficiente entre los contenidos que se entregan en la sala de clases y los materiales de los cuales efectivamente se dispone. Además, en la medida que el maestro logra acercar a sus estudiantes a la biblioteca, ofrece una instancia de entrenamiento en habilidades que les serán transversalmente útiles en otras asignaturas y en la vida en general: la eficacia de la enseñanza es la capacidad de un maestro para inculcar los conocimientos y habilidades en los estudiantes, así como su capacidad para cambiar su comportamiento para vivir mejor.

Sin embargo, para que un profesor logre tener una visión acertada de la conexión que existe entre lo que su biblioteca ofrece y su asignatura, debe ir regularmente a ella y, finalmente, utilizar sus materiales plenamente a la luz de la materia que imparte. Si logra hacer esto, facilitará el proceso de enseñanza-aprendizaje de sus estudiantes, y en ese sentido, será más eficiente.

Tal como señalan Adeoye \& Popoola (2011), el conocimiento de docentes de la materia y sus competencias e ingenio, podrían vincularse a la eficacia de la enseñanza, a la efectividad del maestro y al rendimiento de los estudiantes. Y en la misma línea, un requisito para la eficacia de la enseñanza es un mejor conocimiento de la materia, adquirido a través de la investigación, el uso de biblioteca y de los recursos de información y servicios.

A pesar de que esto pudiera parecer obvio, la verdad es que a menudo los académicos no utilizan plenamente los recursos bibliográficos que se les ofrecen. No obstante, Aiyepeku (1992) nos recuerda que "la supervivencia de un científico en cualquier sistema universitario depende de su capacidad para explotar los recursos de información disponibles" (Cit. en 
Popoola, 2008: en línea). Al respecto existe información variable en cuanto al grado de uso de los recursos de información que la biblioteca les ofrece.

Mientras que Stoan (1991) y Hurych (1986) afirman que sólo un pequeño porcentaje de los científicos sociales usan regularmente recursos de información formales como bases de datos (Cit. en Popoola, 2008), existen datos más recientes que informan un mayor uso por parte de los académicos. Así, por ejemplo, Munster (2003) reporta que un 79\% de los investigadores de las humanidades asiste semanalmente, y un $47 \%$ ocupa el catálogo de búsqueda con la misma frecuencia, existiendo diferencias entre las distintas disciplinas, siendo esto último corroborado por hallazgos en diversos estudios. Kayongo \& Helm (2010) indican que en una muestra de 987 estudiantes de postgrado, un 53,3\% asiste en persona a la biblioteca, mientras que un $65.5 \%$ lo hace desde el hogar. De los que asisten con frecuencia semanal o diariamente, un $34,8 \%$ lo hace para solicitar o devolver un libro.

El hecho de que los profesores incrementen su asistencia (en persona o virtual) a la biblioteca, influye directamente en el conocimiento que tienen de las colecciones disponibles, y de cuánto esas colecciones se relacionan con las materias asociadas a su asignatura. Esta asistencia, sin embargo, se ve afectada por diferentes razones.

Green (2006) realizó un estudio con 404 profesores a través de encuestas y 296 de entrevistas semiestructuradas, encontrando que ocupaban principalmente un motor de búsqueda como Google. Los académicos percibían una serie de obstáculos para el mayor y más efectivo uso de imágenes y colecciones digitales incluyendo herramientas inadecuadas para agregar y administrar imágenes digitales, cuestiones sobre derechos de autor, insuficiente asistencia técnica, falta de tiempo para localizar y aprender a utilizar imágenes digitales y el insuficiente acceso a colecciones.

Hartley et al. (2004, Cit. en McMartin et al., 2008), descubrieron que a menudo los profesores no utilizan y a veces incluso no conocen conceptos como "colección" o "biblioteca digital". Los participantes respondieron que sus principales razones para no usar recursos digitales eran que éstos no apoyaban su enfoque de enseñanza, no tenían tiempo y tenían dificultades para encontrar, administrar, mantener y volver a utilizar material en nuevos contextos. La presencia de la tecnología moderna en bibliotecas contemporáneas, la enorme cantidad de información de recursos, la falta de habilidades necesarias para la utilización eficiente de una biblioteca de investigación, así como las dificultades para interactuar con los bibliotecarios pueden explicar en parte los porcentajes variables de usos de las bibliotecas (Jiao \& Onwuegbuzie, 1997).

Todo esto es crítico en la medida que existe evidencia de que el uso efectivo de los recursos de biblioteca tiene un efecto en la calidad de la enseñanza. Popoola y Haliso (2009) señalan con claridad que existen correlaciones positivas significativas entre la eficacia de la enseñanza y la utilización de recursos de información y los servicios de la biblioteca por parte de los encuestados.

En la Universidad San Sebastián (USS), al igual que en la mayoría de las Universidades, los programas de las asignaturas tienen consignada bibliografía básica y complementaria, que a la vez es la base para la adquisición de textos por parte de la Dirección General de Bibliotecas. Las solicitudes de compra desde las Escuelas se contrastan con los requerimientos bibliográficos del programa de la asignatura y del syllabus de ésta, según el número de alumnos estimados. Con respecto a esto último, jamás se debe perder de vista que el syllabus no es sólo una planificación o un contrato, sino que es una vía para proveer información al estudiante de cómo aprender más efectivamente (Parkes \& Harris, 2002). 
Estudios Pedagógicos XL, N²: 189-201, 2014

EVALUACIÓN DE LA EFECTIVIDAD DE UN PROGRAMA PILOTO DE OPTIMIZACIÓN DEL USO DE LOS RECURSOS DE BIBLIOTECA EN LA UNIVERSIDAD SAN SEBASTIÁN Y DE LA SATISFACCIÓN DE LOS ESTUDIANTES,...

Existe un esfuerzo permanente y creciente de la biblioteca USS por adquirir los textos que las diferentes Escuelas requieren para dictar sus asignaturas. Sólo en el año 2011, la inversión en libros alcanzó \$406.227.163 (Universidad San Sebastián, 2011a). A esto se suma que dentro del proyecto educativo de la USS y el ambiente denominado "Vive la Experiencia USS", se ha establecido como un proyecto importante instalar la cultura de la información científica entre los docentes y bibliotecólogos en el aula como parte del proceso enseñanza-aprendizaje, proporcionándole al estudiante mayor autonomía en la búsqueda de la información, la posibilidad de conocer y aplicar normas bibliográficas, y evaluar la información que utiliza en su trabajo académico (De la Vega y Fukushi, 2011). A esto se le ha denominado proyecto "Cultura de la Información en el Aula". El proyecto "Vive la Experiencia USS", por su parte, busca construir un "ecosistema de aprendizaje" dotado de una serie articulada de dispositivos que, funcionando de manera armónica, favorecen en los estudiantes una mayor velocidad en los avances de los aprendizajes.

Si bien se podría suponer que la existencia creciente de textos en biblioteca asociados a las asignaturas y los dispositivos descritos deberían redundar en una mayor satisfacción de los alumnos con los textos disponibles, esto no ocurre. En dos estudios consecutivos de Calidad de Servicio realizados en la Universidad San Sebastián, la disponibilidad de textos en biblioteca para las asignaturas ha sido calificado de forma deficitaria en escala de 1 a 7 : en el año 2010 con nota 4,8 (Universidad San Sebastián, 2010), y en año 2011 con nota 4,7 (Universidad San Sebastián, 2011b).

Esto podría ocurrir por múltiples razones, entre ellas el hecho de que muchos de los académicos evaluados indirectamente por los estudiantes son profesores part-time, lo cual impacta en la utilización de los recursos y la administración efectiva y válida de las bibliografías. Se ha demostrado que este tipo de docentes exhiben diferencias con los profesores de planta en estos aspectos (Washington-Hoagland \& Clougherty, 2002). Además, si bien todos los profesores reciben un proceso de inducción a la Universidad por parte de los directivos de la Facultad, existen diferencias en las percepciones de los distintos directivos y administradores de una universidad con respecto a la interacción real que debe existir con la biblioteca y el uso pleno de los recursos existentes (Stebelman et al., 1999).

Es así como un académico puede asignar para una evaluación ciertas lecturas que no se encuentran en biblioteca, entregando ejemplares o fotocopias de su uso personal. Al hacer esto transmite al estudiante la imagen de que la colección de biblioteca no sirve a los propósitos educativos de su asignatura: el estudiante llega a final de semestre con insatisfacción con los recursos disponibles en biblioteca. Así, esta insatisfacción no es producto de una inconsistencia entre el programa de la asignatura y lo disponible, sino que más bien, entre las lecturas que el profesor entrega y lo disponible en biblioteca. Esto es grave a la luz de lo señalado por Lau (2001) y por Deborah (2009), quienes indican que una meta básica de un curso de inducción para académicos debería ser alentar a los estudiantes a incluir materiales de la biblioteca en sus estrategias de aprendizaje.

El cambio que debe ocurrir en el académico para una utilización efectiva de los recursos de biblioteca y la subsecuente satisfacción de los estudiantes con los recursos disponibles, es de naturaleza actitudinal: implica que en la sala de clases el académico no sólo sepa claramente las conexiones entre los contenidos y los recursos de biblioteca, sino que además modele su uso eficiente a través de un esfuerzo permanente por asignar textos que se encuentren disponibles para el alumno en biblioteca. 


\subsection{FORMULACIÓN DEL PROBLEMA}

Existe una subutilización y una insatisfacción con los recursos de biblioteca, a partir de una inconsistencia entre los textos consignados en los programas de asignatura, los syllabus y la existencia de materiales en biblioteca, y aquellos efectivamente recomendados por el profesor en la sala de clases.

\section{MÉTODOS}

\subsection{OBJETIVO}

Aumentar el grado de satisfacción con los recursos bibliográficos disponibles en biblioteca mediante la implementación de un cambio actitudinal en los profesores de asignaturas para la asignación de textos.

\subsection{HIPÓTESIS}

Los alumnos de las asignaturas participantes en el programa piloto Claraboya, tendrán un nivel de satisfacción con los recursos disponibles en Biblioteca estadísticamente superior que los alumnos de la Universidad participantes en las encuestas Calidad de Servicio 2010 y 2011.

\subsection{DISEÑO}

El diseño propuesto corresponde al tipo cuasiexperimental, con intervención experimental, posprueba y grupos intactos (Hernández, Fernández y Baptista, 2010). Los docentes de las asignaturas participantes en el proyecto asistieron al denominado Seminario Claraboya, en que se les capacitó en la optimización del uso de los recursos de biblioteca a través de la conexión con los contenidos de sus asignaturas.

\subsection{INTERVENCIÓN}

La intervención consistió en el denominado "Seminario Claraboya: Optimización de uso de bibliografía y bases de datos electrónicas disponibles en biblioteca mediante la implementación de indexación digital en las asignaturas", dirigido a profesores part-time, y cuyo objetivo general fue desarrollar estrategias de optimización del uso de los recursos de biblioteca con fines pedagógicos y académicos mediante el cambio actitudinal (Steintert et al., 2006). Esto, en el contexto del ambiente "Vive la Experiencia" ya descrito.

El seminario al cual asistieron 20 profesores, duró 5 horas cronológicas presenciales (27 de Julio de 2012) y 15 horas cronológicas en total, y tuvo como requisito de aprobación dos componentes:

1) Un trabajo grupal donde expusieron acerca de un tema disciplinar en Power Point que consideró lo siguiente:

- Vinculación de los contenidos expuestos y presentes en sus asignaturas, con bibliografía de especialidad física y virtual disponible en el sistema de bibliotecas de la USS. 
EVALUACIÓN DE LA EFECTIVIDAD DE UN PROGRAMA PILOTO DE OPTIMIZACIÓN DEL USO DE LOS RECURSOS DE BIBLIOTECA EN LA UNIVERSIDAD SAN SEBASTIÁN Y DE LA SATISFACCIÓN DE LOS ESTUDIANTES,...

- Conexión con el syllabus, programa y textos efectivamente sugeridos.

- Modelamiento de conductas orientadas a la utilización efectiva de los recursos de biblioteca.

- Modelamiento de la incorporación de la biblioteca en el aula.

2) Participación en un programa de seguimiento personalizado durante el semestre, en el que se asistió a los veinte (20) profesores en todas aquellas dudas y dificultades que pudieran tener con la implementación de los contenidos en sus asignaturas. Este programa de seguimiento se desarrolló en dos períodos, el primero en una modalidad mixta telefónica/correo electrónico, y el segundo mediante una encuesta online.

Se debe señalar que en dichos seguimientos se verificó el alto nivel de satisfacción con el seminario: la calidad académica de éste y la importancia de los temas expuestos fueron evaluadas con nota 6,4 (en escala de 1 a 7). Así mismo, un 80\% de los asistentes reportaron haber implementado los contenidos en alguna medida a los tres meses de evaluación.

El seminario contó entre otros aspectos con apoyo audiovisual, préstamo de computadores a los académicos para la preparación de las presentaciones e ingreso a catálogo y bases de datos de biblioteca, certificación por parte de la Universidad firmada por las Vicerrectorías involucradas en el proyecto piloto. Ellas fueron la Vicerrectoría Académica, la Vicerrectoría de Sede Concepción y la Vicerrectoría de Aseguramiento de la Calidad de la Universidad San Sebastián. Los expositores fueron la Directora General de Bibliotecas USS, la Bibliotecóloga referencista de la Sede Concepción, el Director de Estudios de la Vicerrectoría Académica y el Director de Evaluación Institucional.

\subsection{MUESTRA}

La muestra por accesibilidad estuvo constituida por 175 estudiantes de las escuelas de Kinesiología, Pedagogía en Educación Diferencial, Trabajo Social y Arquitectura de la Universidad San Sebastián, Sede Concepción. Estos estudiantes cursaron asignaturas con profesores adjuntos, docentes de asignaturas dictadas durante el segundo semestre 2012 en la sede Concepción, participantes del Seminario Claraboya, y que cumplieran los siguientes requisitos: alta solicitud de compra de material bibliográfico en los últimos tres años, que fueran dictadas por profesores adjuntos y con alta rotación de académicos en los últimos tres años.

La muestra de 175 estudiantes estuvo constituida por 117 mujeres y 56 hombres cuya distribución por carrera fue la siguiente: 46 de ellos de Arquitectura (26,4\%), 47 de Kinesiología (27\%), 63 de Pedagogía en Educación Diferencial (36,2\%) y 18 de Trabajo Social (10,3\%).

De las 20 asignaturas originales participantes en el Seminario, contestaron la encuesta estudiantes de 18 de ellas. El listado de asignaturas evaluadas fue el siguiente:

- Análisis de la Arquitectura y Ciudad

- Anatomía II

- Arquitectura y Contexto II

- Ciudad y Contexto II

- Desarrollo de Habilidades Cognitivas

- Diseño Urbano

- Edificación II

- Estructuras II

- Evaluación Psicopedagógica

- Física Médica
- Gestión de Proyectos Matemática Instrumental II

- Intervención Psicopedagógica para el Desarrollo del Lenguaje y del Habla

- Metodologías de Trabajo Social

- Patología en Neurología

- Seminario de Grado

- Taller de Intervención Persona y Familia

- Técnicas Kinésicas de Tratamiento Manual 
Para obtener las muestras Calidad de Servicio 2010 y 2011, se extrajeron aleatoriamente de las bases de datos correspondientes a dichos estudios 175 sujetos de cada una.

Así se cuenta finalmente con tres bases de datos: 175 sujetos de la Encuesta Calidad de Servicio 2010, 175 sujetos de la Encuesta Calidad de Servicio 2011, y 175 sujetos de la medición experimental, grupo EIPC 2012.

\subsection{INSTRUMENTOS}

\subsubsection{Instrumento EIPC}

Para evaluar la efectividad de la intervención, se realizó una adaptación de la Encuesta Calidad de Servicio que la Universidad San Sebastián realiza desde el año 2009. Esta encuesta está dividida en 14 dimensiones, siendo una de ellas biblioteca. La encuesta total ha mostrado una consistencia interna de 0.98 y 0.96 (Alpha de Cronbach) en los años 2011 y 2010, respectivamente.

La sección de biblioteca tiene entre 7 y 9 ítems -dependiendo del año- donde los alumnos asignan nota de 1 a 7 al servicio ahí mencionado. Para los fines del presente estudio, se creó un instrumento denominado Encuesta Investigación Proyecto Claraboya (EIPC), y que fue aplicado en una versión on-line mediante el sistema Surveymonkey. Este instrumento consta de 7 enunciados (Ver Tabla 1), que son reformulaciones de los ítems creados para las Encuestas de Calidad de Servicio 2010 y 2011, pero focalizados en la asignatura que el estudiante cursó.

El estudiante al comienzo de la encuesta marca la carrera que estudia (de las cuatro participantes), y elige una asignatura/profesor para evaluar. De esta forma, una vez contestadas las preguntas sociodemográficas, el estudiante procede a responder los ítems pensando en la asignatura seleccionada previamente, y que aparece nuevamente al comienzo de la página correspondiente.

Tabla 1. Ítems versión Calidad de Servicio y versión EIPC

\begin{tabular}{|l|l|}
\hline Ítems Calidad de Servicio & Ítems Versión EIPC \\
\hline 1. Disponibilidad de Textos en Biblioteca & $\begin{array}{l}\text { Disponibilidad de textos en biblioteca para la asignatura } \\
\text { elegida }\end{array}$ \\
\hline 2. Horario de Biblioteca & $\begin{array}{l}\text { Horario de biblioteca cuando has requerido material } \\
\text { para dicha asignatura }\end{array}$ \\
\hline 3. Servicio de Préstamo de Notebooks & $\begin{array}{l}\text { Servicio de préstamos de notebooks en biblioteca para } \\
\text { trabajos de esta asignatura }\end{array}$ \\
\hline 4. Bases de Datos de Biblioteca & $\begin{array}{l}\text { Bases de datos electrónicas disponibles en biblioteca y } \\
\text { que has utilizado en esta asignatura }\end{array}$ \\
\hline 5. Catálogo de Búsqueda & $\begin{array}{l}\text { Catálogo de búsqueda de materiales para la asignatura } \\
\text { elegida }\end{array}$ \\
\hline 6. Salas de Estudio de Biblioteca & $\begin{array}{l}\text { Disponibilidad de salas de estudio silencioso en } \\
\text { biblioteca (para fines de esta asignatura) }\end{array}$ \\
\hline $\begin{array}{l}\text { 7. Comodidad de las Salas de Estudio en } \\
\text { Biblioteca }\end{array}$ & $\begin{array}{l}\text { Comodidad de salas de estudio silencioso en biblioteca } \\
\text { (cuando las has requerido para la asignatura elegida ) }\end{array}$ \\
\hline
\end{tabular}

Fuente: elaboración propia. 
Estudios Pedagógicos XL, $\mathrm{N}^{\circ}$ 2: 189-201, 2014

EVALUACIÓN DE LA EFECTIVIDAD DE UN PROGRAMA PILOTO DE OPTIMIZACIÓN DEL USO DE LOS RECURSOS DE BIBLIOTECA EN LA UNIVERSIDAD SAN SEBASTIÁN Y DE LA SATISFACCIÓN DE LOS ESTUDIANTES,...

El instrumento EIPC alcanzó en la aplicación definitiva una consistencia interna, medida con Alpha de Cronbach de 0.867.

\subsection{VARIABLES}

- Satisfacción con Recursos de Biblioteca para la Asignatura

Definición Conceptual: Satisfacción con los recursos que biblioteca provee para la asignatura evaluada.

Definición Operacional: Puntaje obtenido en la escala EIPC.

- Satisfacción Servicio de Biblioteca

Definición Conceptual: Satisfacción con los recursos que biblioteca provee para todas las asignaturas.

Definición Operacional: Puntaje obtenido en la dimensión biblioteca, en las Encuestas Calidad de Servicio 2010 y 2011.

\subsection{APLICACIÓN}

La aplicación de la encuesta EIPC se realizó por correo electrónico, mediante el sistema Surveymonkey, entre el 10 diciembre del año 2012 y el 3 de enero del año 2013.

\subsection{ANÁLISIS DE DATOS}

Los análisis de datos se realizaron, en primer lugar, en base a descriptivos para la variable principal. Para evaluar las diferencias entre los puntajes de los sujetos participantes en el estudio y la población general, se seleccionó al azar de los Estudios Calidad de Servicio 2010 y 2011 un número equivalente de sujetos al grupo experimental.

Una vez hecho esto, se compararon las medias de estos tres grupos (Grupo Experimental, Grupo Calidad de Servicio 2010 y Grupo Calidad de Servicio 2011) para evaluar la efectividad del programa, mediante la prueba t de Student.

\section{RESULTADOS}

A continuación, en la Tabla 2, se muestran los puntajes descriptivos para las variables estudiadas, a saber, Satisfacción Servicio de Biblioteca (Calidad de Servicio 2010 y 2011) y Satisfacción con Recursos de Biblioteca para la Asignatura (EIPC).

Tabla 2. Descriptivos para las variables en estudio

\begin{tabular}{|l|c|c|c|c|c|}
\hline & Grupo & Promedio & $\begin{array}{c}\text { Desviación } \\
\text { Estándar }\end{array}$ & $\begin{array}{c}\text { Valor } \\
\text { Mínimo }\end{array}$ & $\begin{array}{c}\text { Valor } \\
\text { Máximo }\end{array}$ \\
\hline \multirow{2}{*}{$\begin{array}{l}\text { Disponibilidad de textos } \\
\text { en biblioteca para las } \\
\text { asignaturas }\end{array}$} & 2010 & 4,73 & 1,722 & 1 & 7 \\
\cline { 2 - 6 } & 2011 & 4,64 & 1,885 & 1 & 7 \\
\cline { 2 - 6 } & EIPC & $\mathbf{5 , 2 1}$ & $\mathbf{1 , 7 1 0}$ & $\mathbf{1}$ & $\mathbf{7}$ \\
\hline
\end{tabular}


Estudios Pedagógicos XL, N² 2: 189-201, 2014 EVALUACIÓN DE LA EFECTIVIDAD DE UN PROGRAMA PILOTO DE OPTIMIZACIÓN DEL USO DE LOS RECURSOS DE BIBLIOTECA EN LA UNIVERSIDAD SAN SEBASTIÁN Y DE LA SATISFACCIÓN DE LOS ESTUDIANTES,...

\begin{tabular}{|c|c|c|c|c|c|}
\hline \multirow{3}{*}{ Horario de biblioteca } & 2010 & 5,78 & 1,522 & 1 & 7 \\
\hline & 2011 & 5,78 & 1,628 & 1 & 7 \\
\hline & EIPC & 6,15 & 1,303 & 1 & 7 \\
\hline \multirow{3}{*}{$\begin{array}{l}\text { Servicio de préstamos de } \\
\text { notebooks en biblioteca }\end{array}$} & 2010 & 4,49 & 1,776 & 1 & 7 \\
\hline & 2011 & 5,13 & 1,620 & 1 & 7 \\
\hline & EIPC & 5,37 & 1,661 & 1 & 7 \\
\hline \multirow{3}{*}{$\begin{array}{l}\text { Bases de datos } \\
\text { electrónicas disponibles en } \\
\text { biblioteca }\end{array}$} & 2010 & 5,18 & 1,626 & 1 & 7 \\
\hline & 2011 & 5,14 & 1,681 & 1 & 7 \\
\hline & EIPC & 5,54 & $\mathbf{1 , 5 9 1}$ & 1 & 7 \\
\hline \multirow{3}{*}{ Catálogo de búsqueda } & 2010 & 5,19 & 1,616 & 1 & 7 \\
\hline & 2011 & 5,26 & 1,605 & 1 & 7 \\
\hline & EIPC & 5,40 & 1,646 & 1 & 7 \\
\hline \multirow{3}{*}{$\begin{array}{l}\text { Disponibilidad de salas de } \\
\text { estudio }\end{array}$} & 2010 & 3,84 & 1,914 & 1 & 7 \\
\hline & 2011 & 4,23 & 2,064 & 1 & 7 \\
\hline & EIPC & 4,44 & 1,909 & 1 & 7 \\
\hline \multirow{3}{*}{$\begin{array}{l}\text { Comodidad de salas de } \\
\text { estudio }\end{array}$} & 2010 & 4,56 & 1,787 & 1 & 7 \\
\hline & 2011 & 4,72 & 1,974 & 1 & 7 \\
\hline & EIPC & 5,62 & 1,626 & 1 & 7 \\
\hline
\end{tabular}

Fuente: elaboración propia.

Como se puede observar, los promedios obtenidos en la medición 2012 EIPC son superiores a los promedios 2010 y 2011 en todas las variables analizadas: Disponibilidad de textos en biblioteca $(2010=4,73 ; 2011=4,64 ;$ EIPC $=5,21)$, Horario de Biblioteca $(2010=5,78 ; 2011=5,78 ;$ EIPC $=6,15)$, Servicio de préstamo de notebooks $(2010=4,49$; 2011=5,13; EIPC=5,37), Bases de datos electrónicas disponibles $(2010=5,18 ; 2011=5,14$; EIPC=5,54), Catálogo de búsqueda $(2010=5,19 ; 2011=5,26 ; \mathrm{EIPC}=5,40)$, Disponibilidad de salas de estudio $(2010=3,84 ; 2011=4,23 ; \mathrm{EIPC}=4,44)$, y Comodidad de salas de estudio $(2010=4,56 ; 2011=4,72 ; \mathrm{EIPC}=5,62)$.

Para verificar si las diferencias estadísticas existentes entre los puntajes obtenidos en las diferentes mediciones son estadísticamente significativas, se llevó a cabo la prueba t de Student para variables independientes. En la siguiente tabla se muestran dichos resultados:

Tabla 3. Resultados de la prueba t de Student para muestras independientes para las variables en estudio

\begin{tabular}{|l|c|c|c|c|c|c|c|c|c|c|}
\hline & \multicolumn{4}{|c|}{ 2010-EIPC } & \multicolumn{5}{c|}{ 2011-EIPC } \\
\hline & Valor t & Gl & Sig. & $\begin{array}{c}\text { Tamaño } \\
\text { del efecto }\end{array}$ & Cohen & Valor t & Gl & Sig. & $\begin{array}{c}\text { Tamaño } \\
\text { del efecto }\end{array}$ & Cohen \\
\hline
\end{tabular}


Estudios Pedagógicos XL, N²: 189-201, 2014

EVALUACIÓN DE LA EFECTIVIDAD DE UN PROGRAMA PILOTO DE OPTIMIZACIÓN DEL USO DE LOS RECURSOS DE BIBLIOTECA EN LA UNIVERSIDAD SAN SEBASTIÁN Y DE LA SATISFACCIÓN DE LOS ESTUDIANTES,...

\begin{tabular}{|l|l|l|l|l|l|l|l|l|l|l|}
\hline $\begin{array}{l}\text { Disponibilidad } \\
\text { de textos en } \\
\text { biblioteca para } \\
\text { las asignaturas }\end{array}$ & $-2,58$ & 329 & $0,01 * * 1$ & 0,11 & $-0,22$ & $-2,89$ & 330 & $0,00 * *$ & 0,11 & $-0,22$ \\
\hline $\begin{array}{l}\text { Horario de } \\
\text { biblioteca }\end{array}$ & $-2,38$ & 333 & $0,02 * *$ & 0,11 & $-0,21$ & $-2,30$ & 337 & $0,02 * *$ & 0,12 & $-0,25$ \\
\hline $\begin{array}{l}\text { Servicio de } \\
\text { préstamos de } \\
\text { notebooks en } \\
\text { biblioteca }\end{array}$ & $-4,48$ & 308 & $0,00^{* *}$ & 0,22 & $-0,45$ & $-1,31$ & 321 & 0,19 & 0,07 & $-0,14$ \\
\hline $\begin{array}{l}\text { Bases de datos } \\
\text { electrónicas } \\
\text { disponibles en } \\
\text { Biblioteca }\end{array}$ & $-1,89$ & 296 & 0,06 & 0,05 & $-0,11$ & $-2,08$ & 301 & $0,04 * *$ & 0,12 & $-0,24$ \\
\hline $\begin{array}{l}\text { Catálogo de } \\
\text { búsqueda }\end{array}$ & $-1,13$ & 312 & 0,26 & 0,05 & $-0,11$ & $-0,79$ & 320 & 0,42 & 0,04 & $-0,09$ \\
\hline $\begin{array}{l}\text { Disponibilidad } \\
\text { de salas de } \\
\text { estudio }\end{array}$ & $-2,83$ & 323 & $0,00^{* *}$ & 0,11 & $-0,22$ & $-0,94$ & 328 & 0,34 & 0,05 & $-0,1$ \\
\hline $\begin{array}{l}\text { Comodidad de } \\
\text { salas de estudio }\end{array}$ & $-5,53$ & 320 & $0,00 * *$ & 0,29 & $-0,61$ & $-4,45$ & 321 & $0,00 * *$ & 0,24 & $-0,49$ \\
\hline
\end{tabular}

Fuente: elaboración propia.

En la comparación 2010/EIPC, se encuentran diferencias estadísticamente significativas en cinco de las cuatro variables estudiadas a favor de EIPC: disponibilidad de textos $(\mathrm{p}=0,01)$, horario de biblioteca $(\mathrm{p}=0,02)$, servicio de préstamos de notebooks $(\mathrm{p}=0,00)$, disponibilidad de salas de estudio $(\mathrm{p}=0,00)$, y comodidad de salas de estudio $(\mathrm{p}=0,00)$. Bases de datos y catálogo de búsqueda no mostraron diferencias significativas.

Por otra parte, y tal como se observa en la tabla anterior, en la comparación 2011/EIPC, se constataron diferencias estadísticamente significativas a favor de EIPC en: disponibilidad de textos $(\mathrm{p}=0,11)$, horario de biblioteca $(\mathrm{p}=0,12)$, bases de datos disponibles en biblioteca $(\mathrm{p}=0,04)$, y comodidad de salas de estudio $(\mathrm{p}=0,00)$.

Con respecto al tamaño del efecto, se debe indicar que en ambos grupos de comparaciones, todas aquellas que mostraron significancia en la comparación de medias presentaron un tamaño del efecto pequeño medido con la d de Cohen (cercano a 0,2 - 0,3), con excepción de la comodidad de las salas de estudio que presentó en la comparación 2011/EIPC un tamaño del efecto medio (cercano a 0,5).

\section{ANÁLISIS Y DISCUSIÓN}

La presente investigación es pionera en Chile en la evaluación de un programa piloto de optimización del uso de los recursos de biblioteca. Se encontró un mayor nivel de 
satisfacción en todos los indicadores generados y medidos en el año 2012 mediante la escala EIPC. De ellos se encontró que 5 de estos indicadores presentaron un aumento significativo en relación al año 2010, y en 5 indicadores en la comparación con el año 2011. Esto se traduce en una comprobación sustantiva de la hipótesis de investigación.

$\mathrm{Al}$ analizar en detalle esta variación, se detecta que el indicador más importante para los fines de esta investigación, la relación entre "disponibilidad de textos para las asignaturas" y "disponibilidad de textos para esta asignatura", presenta un aumento estadísticamente significativo de 0,49 puntos en relación al año 2010, y 0,57 puntos en relación al año 2011. Si se considera que las muestras 2010 y 2011 fueron seleccionadas de forma aleatoria, estos resultados son positivos para los autores de la presente investigación, y en sí mismos avalan la utilización del programa Claraboya para los objetivos que fue diseñado.

Como ya se mencionó, a esto se suman otros 4 indicadores con variación significativa en la comparación con el año 2010, y 5 indicadores en la comparación con el 2011. De ellos, el aumento en los niveles de satisfacción con el horario de biblioteca puede haberse producido a partir de un círculo virtuoso: en la medida que el estudiante asiste con más frecuencia a buscar textos a biblioteca, se familiariza y adecúa más plenamente a dichos horarios.

El hecho de que la satisfacción con el catálogo de búsqueda no presentara un aumento tras la intervención en ninguno de los dos años, se debe -desde el punto de vista de los autores de este estudio- a que los alumnos de las asignaturas experimentales no se vieron necesariamente estimulados a su utilización por los académicos, en la medida que los títulos eran entregados, en muchos casos, incluso con el código necesario para solicitarlo en el mesón de atención.

La situación variable de las bases de datos (ítem 4), el uso de notebooks (ítem 3) y de salas de estudio (ítems 6 y 7), se debe analizar caso a caso debido a las características idiosincráticas de cada carrera y asignatura. Por ejemplo: un alumno de una carrera de ciencias de la salud probablemente estará más familiarizado con el uso de bases de datos electrónicas de artículos en inglés o, en otro ejemplo, no necesariamente encontrar los textos para la asignatura en biblioteca influye en el uso que se le da a los computadores portátiles que biblioteca ofrece.

Esto último ya señala un aspecto interesante y susceptible de ser profundizado en futuras investigaciones. Hecho este análisis por carrera, avanzar en un estudio análogo al expuesto pero con un diseño experimental, o comparar la efectividad de la intervención dentro de un mismo año con asignaturas no expuestas al programa, se evidencian como limitaciones del estudio y desafíos hacia el futuro para los autores.

Más allá de estas especificidades, desde el punto de vista de los autores se puede afirmar con suficiente certidumbre que el proyecto funciona, debido a que exige a los académicos no sólo conocer los recursos que el sistema de bibliotecas les ofrece, sino que modelar en la sala de clases y con los estudiantes su vinculación con los contenidos expuestos.

Finalmente, la efectividad de los programas orientados al cambio conductual mediante el cambio actitudinal en los docentes universitarios se encuentra suficientemente documentado en la investigación en educación superior (Dierking \& Fox, 2013; Kaplan, Cook \& Steiger, 2006; Khan \& Pred, 2002) en términos de la satisfacción de los académicos y la enseñanza que entregan, pero no en la misma medida en términos de la satisfacción de los estudiantes o del desarrollo organizacional (Steintert et al., 2006). En este sentido, el presente estudio es un aporte para futuros desarrollos en el área. 
Estudios Pedagógicos XL, N²: 189-201, 2014

EVALUACIÓN DE LA EFECTIVIDAD DE UN PROGRAMA PILOTO DE OPTIMIZACIÓN DEL USO DE LOS RECURSOS DE BIBLIOTECA EN LA UNIVERSIDAD SAN SEBASTIÁN Y DE LA SATISFACCIÓN DE LOS ESTUDIANTES,...

\section{REFERENCIAS BIBLIOGRÁFICAS}

Adeoye, M. O. \& Popoola, S. O. (2011). Teaching Effectiveness, Availability, Accessibility, and Use of Library and Information Resources among Teaching Staff of Schools of Nursing in Osun and Oyo State, Nigeria. Library Philosophy and Practice, 1-19. Recuperado de http://search.proquest. com/docview/900093239? accountid=26111, consultado en enero de 2013.

De la Vega, R. y Fukushi, K. (2011). Plan de Requerimientos "Vive la Experiencia USS" (1 Ed.). Santiago: Ediciones Universidad San Sebastián.

Deborah, L. P. (2009). Influencing the Now and Future Faculty: Retooling Information Literacy. Music Library Association, vol. 66, n. 2, 233-248.

Dierking, R. \& Fox, R. (2013). "Changing the Way I Teach": Building Teacher Knowledge, Confidence, and Autonomy. Journal of Teacher Education, vol. 64, n. 2, 129-144.

Green, D. (2006). Using Digital Images in Teaching and Learning: Perspectives from Liberal Arts Institutions. United States: Academic Commons. Recuperado de http://www.academiccommons. org/files/image-report.pdf, consultado en enero de 2013.

Hernández, R., Fernández, C. y Baptista, P. (2010). Metodología de la Investigación (5ª Ed.). México D.F.: Mc-Graw Hill.

Hurych, J. (1986). After Bath: Scientists, Social Scientists and Humanities in the Context of Online Searching. Journal of Academic Librarianship, vol. 12, n. 3, 158-165.

Kayongo, J. \& Helm, C. (2010). Graduate Students and the Library: A Survey of Research Practices and Library Use at the University of Notre Dame. Reference \& User Services Quarterly, vol. 49, n. 4, 341-349. Recuperado de http://search.proquest.com/docview/366193811?account $\mathrm{id}=26111$, consultado en enero de 2013.

Kahn, J. \& Pred, R. (2002). Evaluation of a Faculty Development Model for Technology Use in Higher Education for Late Adopters. Computers in the Schools, vol. 18, n. 4, 127-150.

Kaplan, M., Cook, C. E. \& Steiger, J. (2006). Using Theatre to Stage Instructional and Organizational Transformation. Change: The Magazine of Higher Learning, vol. 38, n. 3, 32-39.

Jiao, Q. \& Onwuegbuzie, A. (1997). Factors Associated with Library Anxiety. Paper presented at the Annual American Educational Research Association Conference. Chicago, IL.

Lau, J. (2001). Faculty-librarian Collaboration: A Mexican Experience. Reference Services Review, vol. 29, n. 2, 95-105.

McMartin, F., Iverson, E., Wolf, A., Morrill, J., Morgan, G. \& Manduca, C. (2008). The Use of Online Digital Resources and Educational Digital Libraries in Higher Education. International Journal on Digital Libraries, vol. 9, n. 1, 65-79.

Munster, I. (2003). La Biblioteca Max von Buch de la Universidad de San Andrés y sus investigadores: Segunda parte de un estudio de las necesidades de información, hábitos y características de Investigadores en Humanidades y Ciencias Sociales. Información, Cultura y Sociedad, n. 9, 53-73.

Parkes, J. \& Harris, M. (2002). The Purposes of a Syllabus. College Teaching, vol. 50, n. 2, 55-61.

Popoola, S. \& Haliso, Y. (2009). Use of Library Information Resources and Services as Predictor of the Teaching Effectiveness of Social Scientists in Nigerian Universities. African Journal of Library, Archives and Information Science, vol. 19, n. 1, 165-77.

(2008). The Use of Information Sources and Services and Its Effect on the Research

Output of Social Scientists in Nigerian Universities. Library Philosophy and Practice, 183. Recuperado de http://unllib.unl.edu/LPP/popoola.html, consultado en enero de 2013.

Stebelman, S., Siggins, S., Nutty, D. \& Long, C. (1999). Improving Library Relations with the Faculty and University Administrators: The Role of the Faculty Outreach Librarian. College \& Research Libraries, vol. 60, n. 2, 121-130.

Steinert, Y., Mann, K., Centeno, A., Dolmans, D., Spencer, J., Gelula, M. \& Prideaux, D. (2006). A Systematic Review of Faculty Development Initiatives Designed to Improve Teaching Effectiveness in Medical Education: BEME Guide No. 8. Medical Teacher, vol. 28, n. 6, 497-526. 
EVALUACIÓN DE LA EFECTIVIDAD DE UN PROGRAMA PILOTO DE OPTIMIZACIÓN DEL USO DE LOS RECURSOS DE BIBLIOTECA EN LA UNIVERSIDAD SAN SEBASTIÁN Y DE LA SATISFACCIÓN DE LOS ESTUDIANTES,...

Stoan, S. K. (1991). Research and Information Retrieval among Academic Researchers: Implications for Library Instruction. Library Trends, vol. 39, n. 3, 238-258.

Washington-Hoagland, C. \& Clougherty, L. (2002). Faculty and Staff Use of Academic Library Resources and Services: A University of Iowa Libraries' Perspective. Libraries and the Academy, vol. 2, n. 4, 627-646.

Universidad San Sebastián. (2011a). Informe de Evaluación Interna. Proceso de renovación de la acreditación institucional. Santiago: Vicerrectoría de Aseguramiento de la Calidad, Universidad San Sebastián.

(2011b). Informe Descriptivo Estudio Calidad de Servicio 2011 Universidad San Sebastián. Dirección de Evaluación Institucional, Vicerrectoría de Aseguramiento de la Calidad. Santiago: Vicerrectoría de Aseguramiento de la Calidad, Universidad San Sebastián. (2010). Informe Descriptivo Estudio Calidad de Servicio 2010 Universidad San Sebastián. Dirección de Evaluación Institucional, Vicerrectoría de Aseguramiento de la Calidad. Santiago: Vicerrectoría de Aseguramiento de la Calidad, Universidad San Sebastián. 
